\title{
A Systematic Review on the Impact of Genetic Testing for Familial Melanoma II: Psychosocial Outcomes and Attitudes
}

\author{
Clare A. Primiero ${ }^{a}$ Tatiane Yanes $^{a} \quad$ Anna Finnane $^{b}$ H. Peter Soyer ${ }^{a} c$ \\ Aideen M. Mclnerney-Leo ${ }^{a}$ \\ aThe University of Queensland Diamantina Institute, The University of Queensland, Dermatology Research \\ Centre, Brisbane, QLD, Australia; ${ }^{b}$ School of Public Health, The University of Queensland, Brisbane, QLD, Australia; \\ 'Department of Dermatology, Princess Alexandra Hospital, Brisbane, QLD, Australia
}

\section{Keywords}

Melanoma - Genetic testing · Psychosocial impact

\begin{abstract}
Background: Although genetic testing for known familial melanoma genes is commercially available, clinical implementation has been restrained as utility is unclear, concerns of causing psychological distress are often cited, and consumer interest and perceptions are not well understood. A review of studies exploring participant-reported psychosocial outcomes and attitudes towards genetic testing for familial melanoma will provide insight into common emotional and cognitive responses. Methods: Database searches of PubMed, Embase, CINAHL, PsycINFO and the Cochrane Library were conducted using a date range of January 1995 to June 2020. Studies examining any psychosocial outcomes alongside genetic testing (real or hypothetical), in participants described as having a high risk of melanoma, were eligible. A narrative synthesis of results was used to describe psychosocial outcomes and summarise participant beliefs and attitudes towards genetic testing. Results: Limited evidence of adverse psychosocial outcomes was found. No impacts on perceived risk or control were reported, and mini-
\end{abstract}

mal decisional regret was recorded. Generalised distress was comparable between both genetic mutation carriers and non-carriers, often decreasing over time from pretesting levels. Melanoma-specific distress was frequently higher in carriers than non-carriers; however, this difference was present prior to testing and often associated with personal melanoma history. Overall, participants' attitudes towards testing were largely positive, with benefits more frequently described than limitations, and support for testing minors was strong. Conclusions: This review has found evidence of few adverse psychological outcomes following genetic testing. There was no indication of increased distress after genetic test results had been disclosed. If these findings were replicated in additional, larger, diverse populations over a longer follow-up period, this would be compelling evidence to guide clinical recommendations.

(c) 2021 S. Karger AG, Basel

\section{Introduction}

It is estimated that $1 \%$ of primary melanoma cases occur within families with a very strong history of melanoma [1]. To date, approximately 10 high-penetrance genes

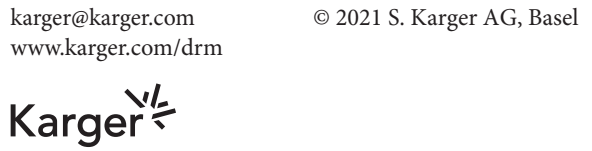

Aideen McInerney-Leo and H. Peter Soyer 
conferring a significantly increased risk of melanoma have been identified; however, they explain only around $22 \%$ of the familial melanoma cases [2]. By far the most common causative gene for familial melanoma is $C D K N 2 A$, a gene implicated in cell cycle regulation [3]. Pathogenic variants in CDKN2A incur a 52\% lifetime risk of melanoma and an increased risk of pancreatic cancer [4]. The penetrance of $C D K N 2 A$ for pancreatic cancer risk is not as well established, with European and North American studies estimating an $18 \%$ lifetime risk $[5,6]$, while Australian cohort studies have found no evidence of increased risk [6]. Possible explanations for the discrepancy may be due to lower smoking rates in Australia and/or differing CDKN2A mutations in Australian populations [6].

Although genetic testing using a panel of known susceptibility genes is commercially available [7], clinical implementation has been restrained as utility in improving prevention is unclear [8]. Additionally, there are concerns about causing psychological distress [9], and a number of complex ethical and public health issues are debated [10]. Previous systematic reviews on predictive genetic testing for various diseases have reported limited evidence of adverse psychosocial impact [11-13], and a modest impact on increasing preventative behaviour [14-16]. There have been no previous systematic reviews focusing solely on outcomes relevant to genetic testing for familial melanoma. This is the second in a 2-part systematic review series, where the first reported impact on preventative behaviour (see part I, the other paper of this series, DOI: 10.1159/000513919), while this review focuses specifically on psychosocial outcomes. This review explores a range of complex psychosocial outcomes beyond psychological distress, including attitudes towards genetic testing and testing of minors, as well as factors influencing uptake.

\section{Methods}

This systematic review was performed according to the Preferred Reporting Items for Systematic Reviews and Meta-Analyses (PRISMA) Statement [17] and was preregistered with PROSPERO (International Prospective Register of Systematic Reviews), and it can be accessed here: https://www.crd.york.ac.uk/prospero/display_record.asp? ID = CRD42020186881.

Inclusion criteria comprised any studies where (i) participants were perceived to be at high-risk of developing melanoma, (ii) genetic testing (or hypothetical scenarios) was offered, and (iii) psychosocial outcomes were available. Only original research published in English was assessed for eligibility. The following databases were searched: Pubmed, Embase, CINAHL, PsycINFO and
Table 1. Glossary of commonly used terms

\begin{tabular}{ll}
\hline $\begin{array}{l}\text { Carrier/ } \\
\text { non-carrier }\end{array}$ & $\begin{array}{l}\text { A person who tests positive or negative for a } \\
\text { familial pathogenic variant (in CDKN2A) }\end{array}$ \\
\hline $\begin{array}{l}\text { Affected/ } \\
\text { unaffected }\end{array}$ & $\begin{array}{l}\text { A person with or without a personal history (of } \\
\text { melanoma) }\end{array}$ \\
\hline Controls & $\begin{array}{l}\text { Participants who received counselling without } \\
\text { genetic testing }\end{array}$ \\
\hline
\end{tabular}

the Cochrane Library using terms related to genetic testing, psychosocial impact and melanoma risk; search strings are provided in online supplementary Table S1 (for all online suppl. material, see www.karger.com/doi/10.1159/000513576). A date range of January 1995 (discovery of CDKN2A as a melanoma gene) until June 2020 was applied. Screening was performed by reviewing titles/abstracts. Eligibility was assessed by reviewing full text. A subsequent backwards and forwards citation search was completed. The QualSyst assessment tool [18] was used to review the quality of studies included in the review. Screening, eligibility, quality and risk assessment was performed by author C.P., and a second author (T.Y.) screened $10 \%$ of the records and assessed quality in $25 \%$. Data were extracted using a standardised report form, collecting: citation, study design, study population, outcomes and follow-up time points. Narrative synthesis was used to describe results, as the differences in methods and outcomes measured prevented result pooling [19].

A glossary of commonly used terms throughout this review is provided in Table 1.

\section{Results}

In total, 2,352 articles were identified in the database and citation search, and 1,697 remained after removing duplicates. A further 1,590 articles were removed after screening titles/abstracts, leaving 49 articles assessed for eligibility by full-text review (Fig. 1). Thirty articles were excluded for reasons described in Figure 1. The final review included 21 articles describing outcomes from 12 unique studies, which included a total of 1,137 study participants. Two studies used hypothetical scenarios to explore psychosocial responses, and the remaining studies used CDKN2A genetic testing to provide genetic risk assessment. No other familial melanoma risk genes were assessed. The follow-up period ranged from cohort studies to 2 years after test result disclosure. Table 2 describes characteristics of studies, including outcomes assessed. Interrater reliability scored highly between authors for screening and quality assessment, producing a kappa score of 0.88 for screening and 0.91 for quality assess- 


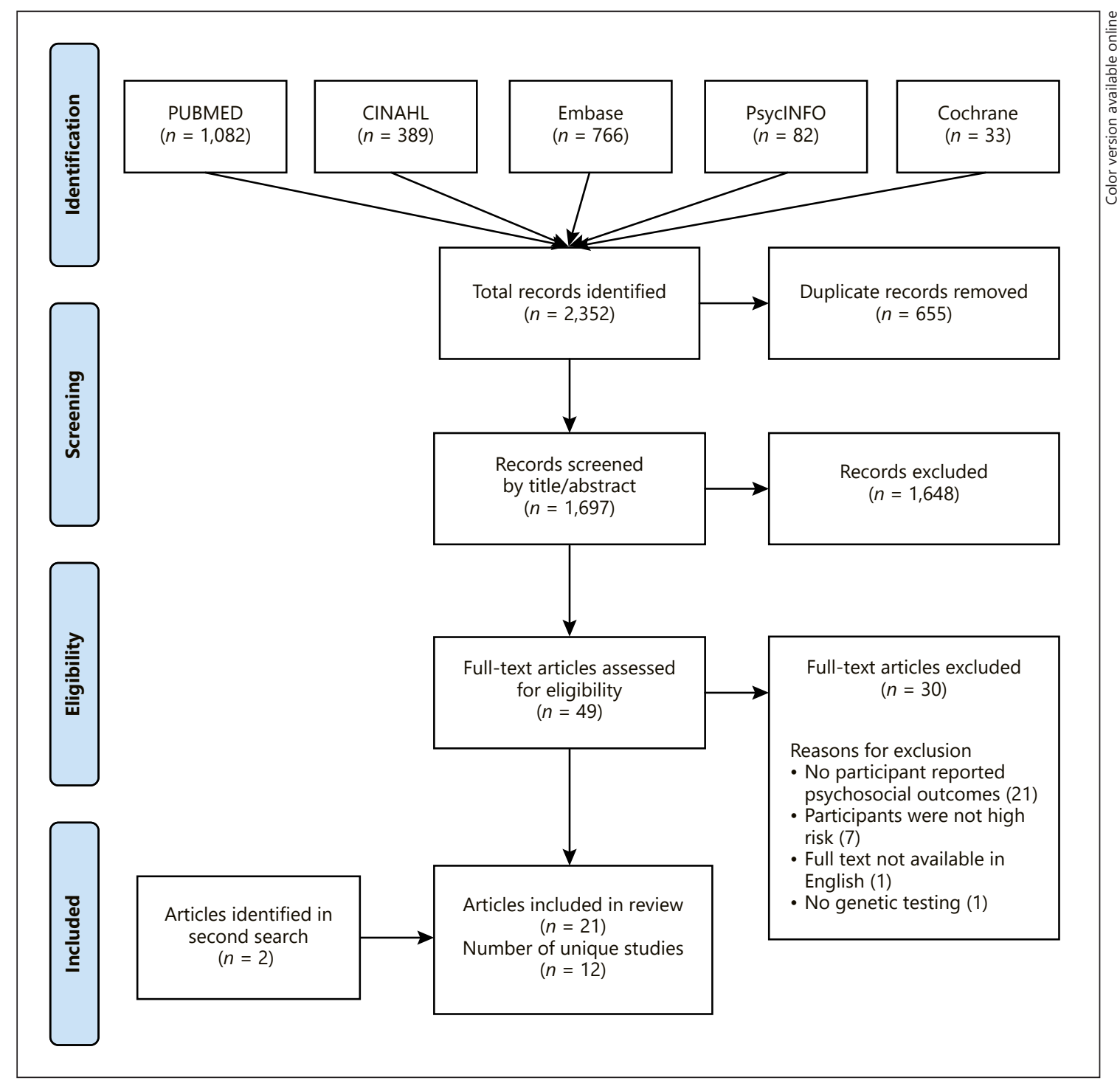

Fig. 1. Flowchart of the database and citation search.

ment. Quality assessment found all studies rated moderate to high in quality (online suppl. Table S2).

\section{Adverse Psychological Outcomes}

The most commonly reported psychological outcomes assessed included: generalised anxiety and depression [20-26]; melanoma-specific distress [20-28]; cancer (melanoma and pancreatic) worry/concern [24, 26-28]; and decision (for genetic testing) regret [20, 2426]. Most studies utilised validated, widely accepted surveys such as the Hospital Anxiety and Depression Scale which includes two 7-item subscales to measure both anxiety and depression, and is widely used in research
[29]; the Impact of Events Scale, which comprises 15 items to assess intrusive and avoidant cognitions and behaviours in respondents and has been used to measure disease-specific distress [30]; and the Multidimensional Impact of Cancer Risk Assessment scale, a 25-item survey that is commonly used to assess distress and uncertainty in people undergoing genetic testing [31]. Other validated questionnaires were used to capture cancer worry [32-34], decision regret [35], mental health [36], anxiety and depression in adolescents $[37,38]$, and lastly open-ended and semi-structured interviews were used in 3 studies $[25,26,28]$. 


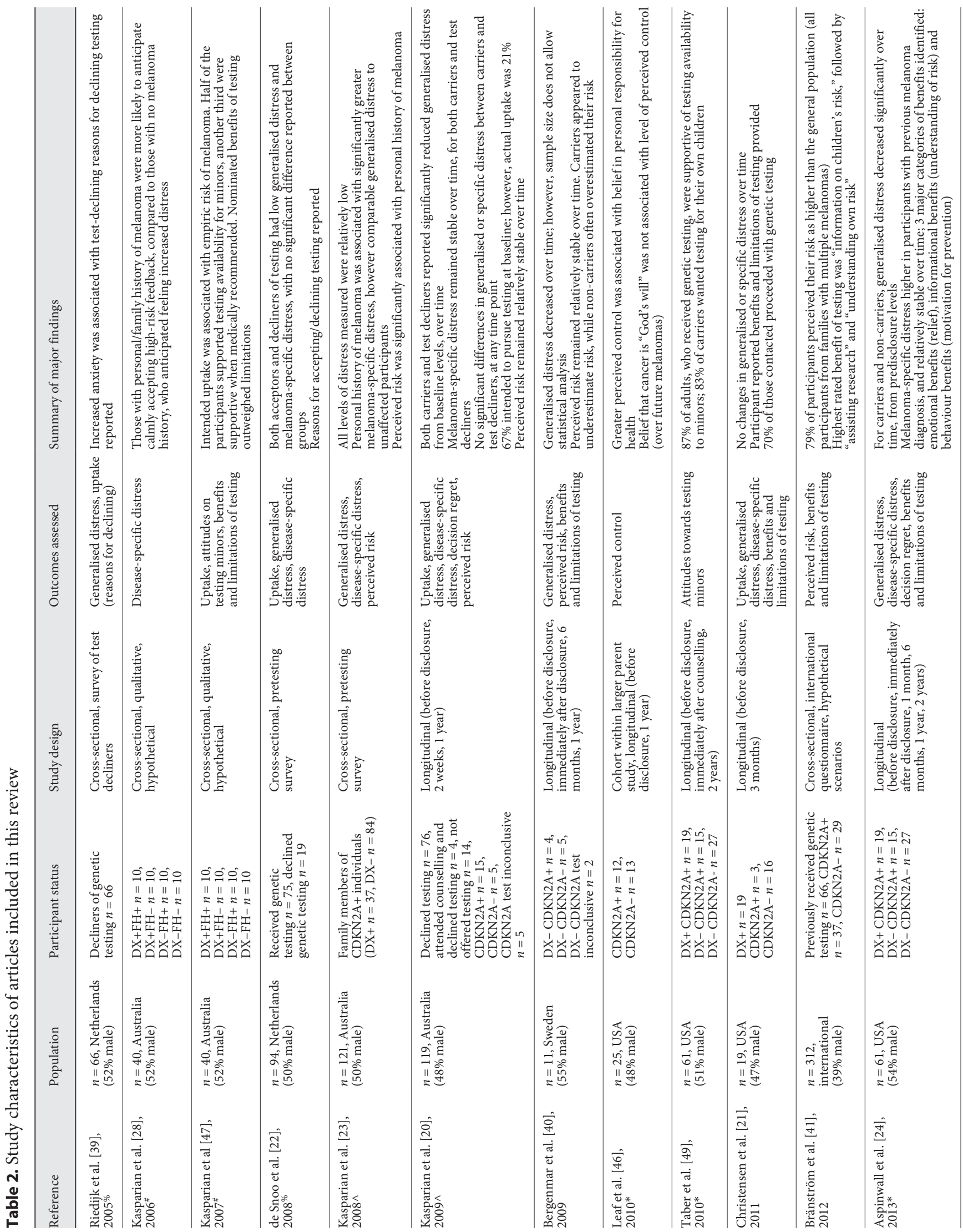




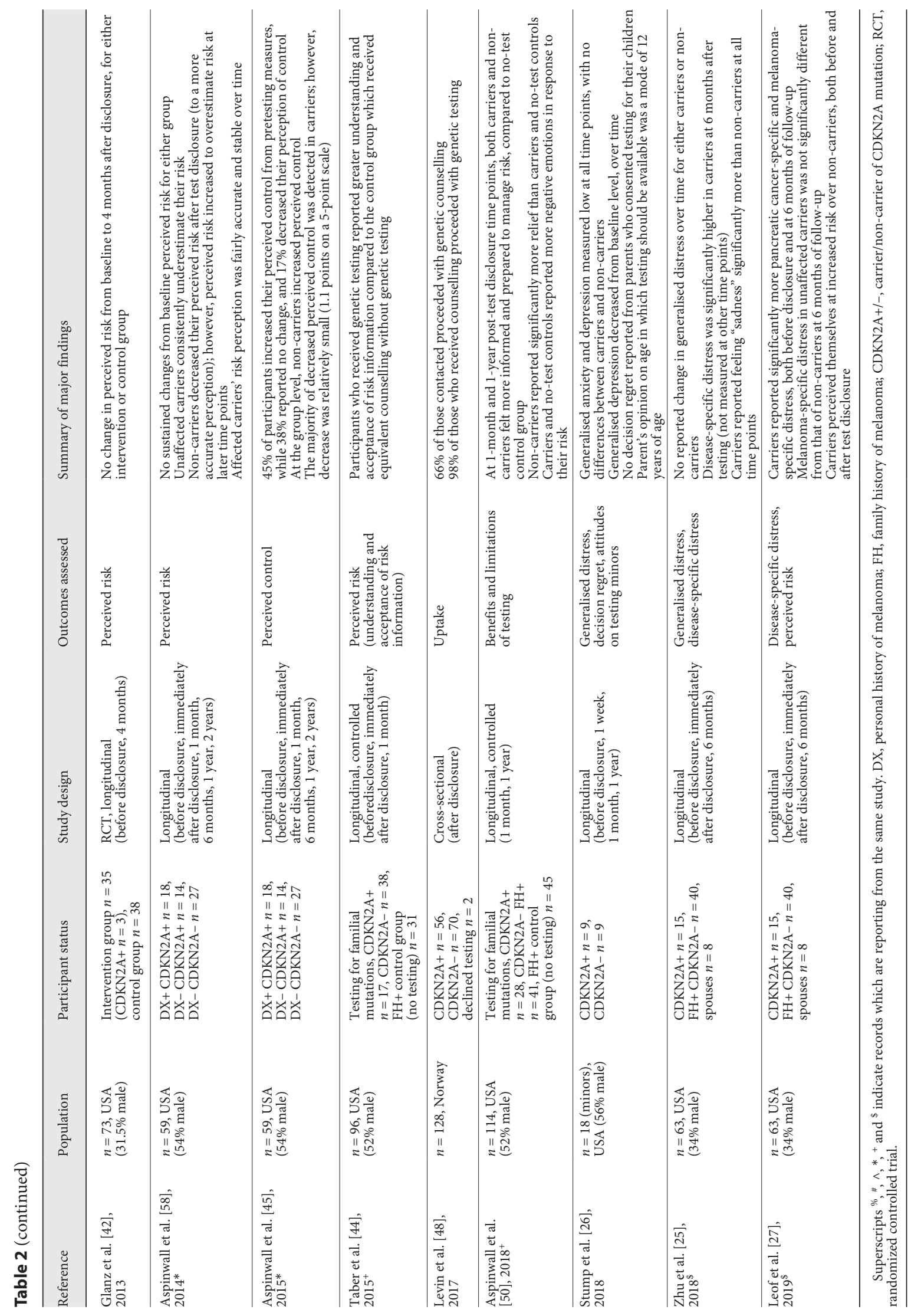


Generalised Distress (Anxiety and Depression)

Generalised distress was reported in 9 publications from 7 separate studies [20-26, 39, 40]. All studies used validated measures to quantitatively assess outcomes related to generalised anxiety and depression [20-26, 39, 40]. Within studies, results were compared between a variety of subgroup populations, such as mutation carriers, non-carriers, test acceptors, test decliners, those previously diagnosed with melanoma (affected) and those without (unaffected). Across all studies, mean distress scores were low and below clinically relevant thresholds. No studies reported an increase from baseline measures in generalised anxiety or depression following test disclosure. Four studies reported no significant change in anxiety in carriers or non-carriers over time $[21,25,26,40]$. One study $(n=119)$ reported no association between generalised distress scores at baseline (before testing) and personal melanoma history [23]. At 12 months after testing, this study measured a significant decrease in anxiety for both acceptors and decliners of testing [20]. Another study $(n=60)$, which compared affected carriers, unaffected carriers and non-carriers, found a significant decrease in anxiety across all groups over time [24]. One cross-sectional study which surveyed participants prior to testing reported low levels of anxiety and depression for both acceptors and decliners of testing; however, anxiety was slightly higher in decliners (mean anxiety score 2.5; SD 2.8) compared to acceptors (mean anxiety score 1.7; SD 1.9) [22, 39]. Two studies reported significant decreases in depression over time, which was not dependent on carrier status $[20,26]$. One study found carriers reported "sadness" levels significantly greater than noncarriers; however, this difference was existing at baseline and remained unchanged at 6 months of follow-up [25].

\section{Disease-Specific Distress and Worry}

Disease-specific distress or worry for melanoma was reported in 8 studies [20-28] and for pancreatic cancer in 3 studies [22, 24, 27].

Overall, melanoma-specific distress was relatively low and stable over time, with no significant changes from before to after genetic testing [20, 21, 24]. Group comparisons in one study found no significant differences in baseline distress between acceptors and decliners of testing [22]. Another study reported no differences in melanoma-specific distress between carriers, test acceptors or test decliners at either baseline or 12 months of follow-up [20]. Three studies reported significantly higher distress in specific subgroups including: affected participants [23], affected carriers [24] and in carriers (both affected and unaffected) [25], with no significant change in distress from pre- to post-testing surveys.

Four studies reported on melanoma-specific worry [24, 26-28]. A study evaluating responses to hypothetical scenarios found that a personal or family history of melanoma was associated with calmly accepting increasedrisk results [28]. In comparison, those without any melanoma history anticipated feeling increasingly worried, scared and/or shocked [28]. Two later studies, which provided genetic testing, also considered personal history and reported that affected carriers had significantly greater worry than unaffected carriers and non-carriers, with no significant change over time [24,27]. A study, which tested 18 minors, reported a significant decrease in melanoma-related worry over time (1 year), for both carriers and non-carriers, with no significant difference between groups [26].

Three studies assessed impact of genetic testing on pancreatic cancer-specific worry. One study reported that approximately half of the participants were both surprised and anxious to learn about pancreatic cancer risk [22]. Another study found that carriers had significantly greater pancreatic cancer worry at both baseline (before testing) and 6 months after disclosure [27]. Similarly, another study found both carriers and those with a pancreatic cancer family history recorded significantly greater worry [24]. Furthermore, mean pancreatic cancer worry scores for all participants increased slightly at 1 month, then decreased significantly from 6 months to 2 years after test disclosure [24].

\section{Perceived Risk and Control}

Ten publications, from 7 unique studies, reported outcomes on participants' perceived risk of melanoma [20, $23,27,40-44]$ and perceived control of subsequent melanomas $[45,46]$. Four longitudinal studies found no sustained significant changes in perceived risk from pre- and post-testing, with follow-up ranging from 4 months to 2 years $[27,40,42,43]$. Carriers and those with a personal history of melanoma reported higher perceived risk compared to non-carriers and unaffected individuals $[23,27$, $41,43]$. A study comparing acceptors and decliners of testing found no significant baseline differences in reported risk perception [23]. When comparing objective risk to perceived risk, one study revealed that on average, carriers underestimated risk, while non-carriers overestimated their risk [40]. Likewise, Aspinwall et al. [43] found that unaffected carriers consistently underestimated their risk, non-carriers overestimated, while affected carriers were more accurate. Furthermore, Aspinwall et al. [43] 
reported that while non-carriers recorded decreased perceived risk immediately after test disclosure, long-term follow-up from 1 month to 2 years found levels returning to previous levels of overestimated risk perceptions. Lastly, one study reported that understanding and acceptance of risk information was higher in those who received genetic testing, compared to those who received equivalent counselling without genetic testing [44].

Two studies reported on perceived control of developing subsequent primary melanoma(s) $[45,46]$. In one study, $45 \%$ of participants reported increased perceived control 2 years after genetic testing, while $38 \%$ reported no change, and $17 \%$ reported decreased perception of control [45]. In the second study, individuals who reported greater personal responsibility over their health were also found to have higher perceived control over melanoma risk [46], while religiosity, including belief that cancer was "God's will," was not associated with perceived control [46].

\section{Decisional Regret}

Minimal decisional regret over uptake of genetic testing was reported in 4 studies [20,24-26]. Using the Decision Regret Scale, Kasparian et al. [20] reported minimal regret among carriers and non-carriers. Out of a possible score of 100 , with higher scores representing great regret, the mean score for carriers was 15, compared to 6 for noncarriers [20]. A study offering testing for minors surveyed participants' mothers with open-ended questions and found no self-reported regret in consenting to testing for their children [26].

\section{Uptake of Genetic Testing}

An early Australian study, using hypothetical testing scenarios, reported $68 \%$ of participants would seek genetic testing should it become available [47]. Intentions to take up testing was greater in participants with higher empiric risk. For example, $90 \%$ of participants with both family and personal history of melanoma would pursue testing if available, compared to $30 \%$ of participants with no melanoma history [47]. Additionally, the study used hypothetical testing scenarios of different $C D K N 2 A$ penetrance models $(100,90,30 \%)$ and found little difference in uptake between 100 and $90 \%$; however, uptake declined significantly in the $30 \%$ penetrance scenario [47].

Four studies reported the percentage of contacted participants who proceeded with genetic testing, which included 21 [20], 66 [48], 70 [21] and 77\% [22]. Kasparian et al. [20] reported that intentions to have genetic testing at baseline were $67 \%$. However, at the conclusion of the study only $21 \%$ of participants had undergone genetic testing [20]. The low uptake of testing was attributed to study design factors including participants required to approach a clinical genetic service themselves to request testing and needing to meet the clinics criteria for publicly funded genetic testing [20].

Two studies reported participants' reasons for declining testing, which included: "happy with life how it is," "not relevant to me" and "can't tell me if I'll definitely get melanoma" [20,39]. Factors positively associated with uptake included: female gender, family history, older age, having children, perceived risk, higher empiric pretest risk, lower pretest anxiety and the gene penetrance [20, $22,39,47,48]$.

\section{Attitudes on Testing Minors}

Three studies reported outcomes on participants' attitudes towards testing minors [26, 47, 49]. When asked about attitudes towards testing minors for familial melanoma, approximately half of the participants (53\%) were supportive generally, a third stipulated only under medical recommendation, and $10 \%$ opposed melanoma genetic testing in minors [47]. A later, quantitative study of adults who received testing for CDKN2A found $87 \%$ expressed support for testing availability for children, with greater support in individuals diagnosed with melanoma at a younger age [49]. Participants' perceived benefits for testing children included raising risk awareness and improving primary and secondary prevention $[26,49]$. The perceived minimum testing age ranged from 0 to 25 years, with 12 being the most common answer [49]. A study which provided genetic testing for 18 minors (mean age of 12.4 years), interviewed parents of participants 1 month and 1 year after testing, and found all mothers rated the experience beneficial, due to promoting risk awareness $(90 \%)$ and increasing sun protection behaviour (82\%) [26].

\section{Perceived Benefits and Limitations of Genetic Testing}

Seven studies provided participant-reported outcomes on potential benefits and limitations of genetic testing for familial melanoma [20,21, 24, 40, 41, 47, 50], 2 of which used only hypothetical scenarios of testing $[41,47]$. Overall, benefits were far more frequently reported than limitations, for example, in one study using open-ended questions, nearly $95 \%$ of participants provided at least one benefit at each assessment, compared to $16 \%$ of all study participants who provided a perceived limitation of testing [24]. Across the studies, the most commonly cited benefits reported included: increased awareness/certainty 
of own risk, learn about children's risk, motivation for sun-protective behaviour and screening, assisting research and relief for non-carriers. The most commonly identified disadvantages included: negative psychosocial reaction, concern about effect on family, possible test inaccuracy, reduced protective behaviour (for non-carriers) and insurance discrimination. Furthermore, 3 studies reported results separately for carriers and non-carriers [24, $40,50]$. Carriers most commonly rated motivational benefits (e.g., for sun protection), while non-carriers rated emotional relief regarding personal and children's risk as most important. Both carriers and non-carriers frequently reported informational benefits. Predictably, increased worry for personal risk and children's risk were often reported by carriers as disadvantages of testing.

\section{Discussion}

Based on our systematic review there is limited evidence that genetic testing for familial melanoma causes significant psychological distress. The evidence across the studies, which are based on well-validated multi-item inventories, indicates that generalised distress does not appear to be impacted by testing outcomes, carrier status or personal history. Although measures of psychological distress across the studies were, on average, relatively low and below clinically relevant thresholds, significant differences were detected between subgroups. Presence of higher pretest anxiety in some studies suggests that the testing process itself may cause a short-term increase in generalised distress, which resolves quickly thereafter. In comparison, disease-specific distress seems associated with carrier status and/or personal history. Quantitative measures show that carriers and those with personal history, on average, report significantly higher levels of disease-specific distress, with little change over time. Elevated disease-specific distress in these groups is present at baseline, prior to testing, and therefore not the result of testing outcomes and possibly due to personal or family history. Incidence of higher disease-specific distress in carriers and those previously diagnosed with melanoma is expected and is potentially important in motivating risk-reducing behaviour. Common psychological constructs predict that while excessive levels of distress may have a detrimental impact on health behaviour uptake, moderate levels of distress promote risk reduction [51].

Somewhat surprisingly, there was no evidence that genetic risk assessment had any impact on participants' perceived risk of subsequent melanomas. While it would ap-

A Systematic Review on the Impact of Genetic Testing for Familial Melanoma II pear illogical that personalised genetic risk feedback would not influence perceived risk, it is however consistent with other multifactorial diseases [16]. One reasoning for unchanging risk perception would be if pre-existing beliefs were accurate. On closer examination, it appears while carriers with a previous melanoma diagnosis held fairly accurate risk perceptions, unaffected carriers more often underestimated their risk. When applied to psychological models on health behaviour such as the Protective Motivation Theory [52], this lack of impact on perceived risk may translate to a lack of adapting riskreducing behaviours. It is important to consider the possible differences in how genetic risk results are disclosed, and how much risk education is provided. This is often not described in detail in studies, and therefore, conclusions on efficacy cannot be drawn. However, future investigation into genetic risk counselling and education methods may be beneficial [53].

When reviewing participants' attitudes and beliefs regarding wider availability of genetic testing for familial melanoma, feedback was largely positive, and participants were also in favour of making testing available to minors. The most frequently nominated benefits included greater awareness of own risk and children's potential risk, and motivation for protective behaviour and screening. For those testing negative for a familial mutation, relief rated highly. Concerns regarding impact on relatives and insurance discrimination were also voiced. Both topics are complex issues and require meaningful discussion in pretest counselling, allowing an informed decision to proceed with testing [53]. Positively, several countries have introduced policies or legislation to protect against the use of genetic test information in medical and/or life insurance underwriting [54].

Measured uptake of genetic testing in research provides some indication of the potential demand from the familial melanoma cohort, if testing became more widely available. It is encouraging to see evidence that intentions to pursue genetic testing appear mostly aligned with empiric risk [47]. This may ease concerns that testing services may be overwhelmed by individuals deemed ineligible for testing. One study reported a much lower test uptake (21\%) [20] than others. Initially, this appears to be an outlier; however, it may actually represent a more realistic model of uptake. In this study, participants were required to arrange testing themselves thereby reflecting real-life practice, compared to other study designs which followed more streamlined protocols. This is consistent with uptake of BRCA1 and BRCA2 testing, which was higher when offered to a highly invested research popula- 
tion (78\%) [55], as compared to clinical cohorts of 30\% in men and $62 \%$ in women [56]. The opportunity to learn more about children's potential risk also rated highly as a motivator for uptake of genetic testing [22]. Reasons for declining testing included those relating to convenience, such as time and travel, and the sentiment "I'm happy with life how it is" [20]. A study from the Netherlands reported an association with generalised anxiety and disease-specific worry with declining genetic testing $[22,39]$; however when measured in an Australian cohort, no difference in distress was reported between test acceptors and decliners [20].

There are limitations to consider for this systematic review. The heterogeneity of study methodology used and the inclusion of hypothetical scenarios to draw participant responses places difficulty in synthesising outcomes and limits subsequent conclusions. However, it was assuring to note that outcomes provided in the hypothetical studies were closely aligned with clinical study outcomes reviewed. Further limitations to generalisability include small, homogenous (white, well-educated) self-selected study populations who were highly motivated to participate in the study, and in some studies included several participants from a small number of families. It would be interesting to see whether studies offering genetic testing to a large number of unrelated individuals from differing socio-economic backgrounds yield similar psychosocial outcomes. The overall low sample sizes and lack of gold standard randomised controlled trials place restrictions on the strength of evidence drawn from this review [57]; however, the low incidence of mutation carriers and ethical issues of controlled randomisation are limiting. Therefore, as this is an emerging field with limited research conducted, the importance of capturing all available outcomes was prioritised, and subsequent conclusions are made with caution. While 5 studies followed participants for 1-2 years, a third of the studies in this review were cross-sectional, and another 3 included a follow-up period of 6 months or less, which limits conclusions regarding the long-term psychosocial consequences of genetic testing in familial melanoma. Lastly, grey literature and conference abstracts were not included in the search strategy, and therefore a publication bias risk exists.

\section{Conclusions}

This systematic review has found limited evidence of adverse psychological impacts of genetic testing or negative effects on risk perception. Study participants' atti- tudes towards and perceptions of genetic testing were positive, with perceived benefits far outweighing perceived disadvantages. If larger studies, with more heterogeneous populations and long-term follow-ups produced comparably positive findings, this would be invaluable in informing future clinical practice.

\section{Key Message}

Evidence suggests that genetic testing for familial melanoma does not result in adverse psychosocial outcomes.

\section{Statement of Ethics}

This is a literature review which did not involve human subject recruitment. Thus, ethical approval was not required.

\section{Conflict of Interest Statement}

H.P.S. is a shareholder of MoleMap NZ Limited and e-derm consult $\mathrm{GmbH}$, and undertakes regular teledermatological reporting for both companies. H.P.S. is a medical consultant for Canfield Scientific Inc., Revenio Research Oy and also a medical advisor for First Derm.

\section{Funding Sources}

C.A.P. is funded by an Australian Government Research Training Programme Scholarship. A.M.M.-L. is funded via an NHMRC ECF APP1158111. H.P.S. holds an NHMRC MRFF Next Generation Clinical Researchers Program Practitioner Fellowship (APP1137127)

\section{Author Contributions}

C.A.P. performed database search, screening, eligibility and quality assessment. T.Y. independently screened $10 \%$ of articles and assessed quality of $25 \%$ of articles. C.A.P. prepared the first draft of the manuscript. All co-authors contributed to writing, provided intellectual input and approved the final version of the paper. 


\section{References}

1 Aoude LG, Wadt KA, Pritchard AL, Hayward NK. Genetics of familial melanoma: 20 years after CDKN2A. Pigment Cell Melanoma Res. 2015 Mar;28(2):148-60.

2 Potrony M, Badenas C, Aguilera P, Puig-Butille JA, Carrera C, Malvehy J, et al. Update in genetic susceptibility in melanoma. Ann Transl Med. 2015 Sep;3(15):210.

3 Lynch HT, Shaw TG. Familial atypical multiple mole melanoma (FAMMM) syndrome: history, genetics, and heterogeneity. Fam Cancer. 2016 Jul;15(3):487-91.

4 Cust AE, Harland M, Makalic E, Schmidt D, Dowty JG, Aitken JF, et al. Melanoma risk for CDKN2A mutation carriers who are relatives of population-based case carriers in Australia and the UK. J Med Genet. 2011 Apr;48(4): 266-72.

5 Vasen H, Ibrahim I, Ponce CG, Slater EP, Matthäi E, Carrato A, et al. Benefit of Surveillance for Pancreatic Cancer in High-Risk Individuals: Outcome of Long-Term Prospective Follow-Up Studies From Three European Expert Centers. J Clin Oncol. 2016 Jun;34(17): 2010-9.

6 Goldstein AM, Chan M, Harland M, Hayward NK, Demenais F, Bishop DT, et al.; Melanoma Genetics Consortium (GenoMEL). Features associated with germline CDKN2A mutations: a GenoMEL study of melanomaprone families from three continents. J Med Genet. 2007 Feb;44(2):99-106.

7 Leachman SA, Lucero OM, Sampson JE, Cassidy $\mathrm{P}$, Bruno W, Queirolo $\mathrm{P}$, et al. Identification, genetic testing, and management of hereditary melanoma. Cancer Metastasis Rev. 2017 Mar;36(1):77-90.

8 Kefford R, Bishop JN, Tucker M, Bressac-de Paillerets B, Bianchi-Scarrá G, Bergman W, et al.; Melanoma Genetics Consortium. Genetic testing for melanoma. Lancet Oncol. 2002 Nov;3(11):653-4.

9 Parens E, Appelbaum PS. On What We Have Learned and Still Need to Learn about the Psychosocial Impacts of Genetic Testing. Hastings Cent Rep. 2019;49(Suppl 1):S2-9.

10 Fulda KG, Lykens K. Ethical issues in predictive genetic testing: a public health perspective. J Med Ethics. 2006 Mar;32(3):143-7.

11 Meiser B. Psychological impact of genetic testing for cancer susceptibility: an update of the literature. Psychooncology. 2005 Dec 14(12):1060-74.

12 Broadstock M, Michie S, Marteau T. Psychological consequences of predictive genetic testing: a systematic review. Eur J Hum Genet. 2000 Oct; 8(10):731-8.

13 Collins RE, Wright AJ, Marteau TM. Impact of communicating personalized genetic risk information on perceived control over the risk: a systematic review. Genet Med. 2011 Apr;13(4):273-7.
14 Hollands GJ, French DP, Griffin SJ, Prevost AT, Sutton S, King S, et al. The impact of communicating genetic risks of disease on riskreducing health behaviour: systematic review with meta-analysis. BMJ. 2016 Mar;352:i1102.

15 Frieser MJ, Wilson S, Vrieze S. Behavioral impact of return of genetic test results for complex disease: systematic review and metaanalysis. Health Psychol. 2018 Dec;37(12): 1134-44.

16 Heshka JT, Palleschi C, Howley H, Wilson B, Wells PS. A systematic review of perceived risks, psychological and behavioral impacts of genetic testing. Genet Med. 2008 Jan;10(1): 19-32.

17 Liberati A, Altman DG, Tetzlaff J, Mulrow C, Gøtzsche PC, Ioannidis JP, et al. The PRISMA statement for reporting systematic reviews and meta-analyses of studies that evaluate healthcare interventions: explanation and elaboration. BMJ. 2009 Jul;339:b2700.

18 Kmet L, Lee R. Standard Quality Assessment Criteria for Evaluating Primary Research $\mathrm{Pa}$ pers from a Variety of Fields AHFMRHTA Initiative 20040213. HTA Initiative. 2004;2.

19 Campbell M, Katikireddi SV, Sowden A, McKenzie JE, Thomson H. Improving Conduct and Reporting of Narrative Synthesis of Quantitative Data (ICONS-Quant): protocol for a mixed methods study to develop a reporting guideline. BMJ. 2018;8:e20064

20 Kasparian NA, Meiser B, Butow PN, Simpson JM, Mann GJ. Genetic testing for melanoma risk: a prospective cohort study of uptake and outcomes among Australian families. Genet Med. 2009 Apr;11(4):265-78.

21 Christensen KD, Roberts JS, Shalowitz DI, Everett JN, Kim SY, Raskin L, et al. Disclosing individual CDKN2A research results to melanoma survivors: interest, impact, and demands on researchers. Cancer Epidemiol Biomarkers Prev. 2011 Mar;20(3):522-9.

22 de Snoo FA, Riedijk SR, van Mil AM, Bergman W, ter Huurne JA, Timman R, et al. Genetic testing in familial melanoma: uptake and implications. Psychooncology. 2008 Aug; 17(8):790-6.

23 Kasparian NA, Meiser B, Butow PN, Simpson JM, Mann GJ. Predictors of psychological distress among individuals with a strong family history of malignant melanoma. Clin Genet. 2008 Feb;73(2):121-31.

24 Aspinwall LG, Taber JM, Leaf SL, Kohlmann W, Leachman SA. Genetic testing for hereditary melanoma and pancreatic cancer: a longitudinal study of psychological outcome. Psychooncology. 2013 Feb;22(2):276-89.

25 Zhu X, Leof ER, Rabe KG, McCormick JB, Petersen GM, Radecki Breitkopf C. Psychological Impact of Learning CDKN2A Variant Status as a Genetic Research Result. Public Health Genomics. 2018;21(3-4):154-63.
26 Stump TK, Aspinwall LG, Kohlmann W, Champine M, Hauglid J, Wu YP, et al. Genetic Test Reporting and Counseling for Melanoma Risk in Minors May Improve Sun Protection Without Inducing Distress. J Genet Couns. 2018 Aug;27(4):955-67.

27 Leof ER, Zhu X, Rabe KG, McCormick JB, Petersen GM, Radecki Breitkopf C. Pancreatic cancer and melanoma related perceptions and behaviors following disclosure of CDKN2A variant status as a research result. Genet Med. 2019 Nov;21(11):2468-77.

28 Kasparian NA, Meiser B, Butow PN, Job RF, Mann GJ. Better the devil you know? High-risk individuals' anticipated psychological responses to genetic testing for melanoma susceptibility. J Genet Couns. 2006 Dec;15(6):433-47.

29 Zigmond AS, Snaith RP. The hospital anxiety and depression scale. Acta Psychiatr Scand. 1983 Jun;67(6):361-70.

30 Horowitz M, Wilner N, Alvarez W. Impact of Event Scale: a measure of subjective stress Psychosom Med. 1979 May;41(3):209-18.

31 Cella D, Hughes C, Peterman A, Chang CH Peshkin BN, Schwartz MD, et al. A brief assessment of concerns associated with genetic testing for cancer: the Multidimensional Impact of Cancer Risk Assessment (MICRA) questionnaire. Health Psychol. 2002 Nov; 21(6):564-72.

32 Cappelli M, Verma S, Korneluk Y, Hunter A, Tomiak E, Allanson J, et al. Psychological and genetic counseling implications for adolescent daughters of mothers with breast cancer. Clin Genet. 2005;67(6):481-91.

33 Lerman C, Daly M, Masny A, Balshem A. Attitudes about genetic testing for breast-ovarian cancer susceptibility. J Clin Oncol. 1994; 12(4):843-50.

34 Lerman C, Trock B, Rimer BK, Jepson C, Brody D, Boyce A. Psychological side effects of breast cancer screening. Health Psychol. 1991;10(4):259-67.

35 Brehaut JC, O'Connor AM, Wood TJ, Hack TF, Siminoff L, Gordon E, et al. Validation of a decision regret scale. Med Decis Making. 2003 Jul-Aug;23(4):281-92.

36 Rumpf HJ, Meyer C, Hapke U, John U. Screening for mental health: validity of the MHI-5 using DSM-IV Axis I psychiatric disorders as gold standard. Psychiatry Res. 2001 Dec;105(3):243-53.

37 Kovacs M. Children's Depression Inventory 2 (CDI 2). North Tonawanda: Multi-Health Systems; 2010.

38 Spielberger C, Edwards C. Preliminary test manual for the State-trait Anxiety Inventory for Children: ("How-I-feel questionnaire"). Palo Alto: Consulting Psychologists Press; 1973.

39 Riedijk SR, de Snoo FA, van Dijk S, Bergman W, van Haeringen A, Silberg S, et al. Hereditary melanoma and predictive genetic testing: why not? Psychooncology. 2005 Sep;14(9): $738-45$.
A Systematic Review on the Impact of Genetic Testing for Familial Melanoma II
Dermatology 2021;237:816-826

DOI: $10.1159 / 000513576$ 
40 Bergenmar M, Hansson J, Brandberg Y. Family members' perceptions of genetic testing for malignant melanoma-a prospective interview study. Eur J Oncol Nurs. 2009 Apr;13(2): 74-80.

41 Bränström R, Kasparian NA, Affleck P, Tibben A, Chang YM, Azizi E, et al. Perceptions of genetic research and testing among members of families with an increased risk of malignant melanoma. Eur J Cancer. 2012 Nov; 48(16):3052-62.

42 Glanz K, Volpicelli K, Kanetsky PA, Ming ME, Schuchter LM, Jepson C, et al. Melanoma genetic testing, counseling, and adherence to skin cancer prevention and detection behaviors. Cancer Epidemiol Biomarkers Prev. 2013 Apr;22(4):607-14.

43 Aspinwall LG, Taber JM, Kohlmann W, Leaf SL, Leachman SA. Perceived risk following melanoma genetic testing: a 2-year prospective study distinguishing subjective estimates from recall. J Genet Couns. 2014 Jun;23(3): 421-37.

44 Taber JM, Aspinwall LG, Stump TK, Kohlmann W, Champine M, Leachman SA. Genetic test reporting enhances understanding of risk information and acceptance of prevention recommendations compared to family history-based counseling alone. J Behav Med. 2015 Oct;38(5):740-53.

45 Aspinwall LG, Stump TK, Taber JM, Kohlmann W, Leaf SL, Leachman SA. Impact of melanoma genetic test reporting on perceived control over melanoma prevention. J Behav Med. 2015 Oct;38(5):754-65.
46 Leaf SL, Aspinwall LG, Leachman SA. God and agency in the era of molecular medicine: religious beliefs predict sun-protection behaviors following melanoma genetic test reporting. Arch Religionspsychol. 2010;32(1): $87-112$.

47 Kasparian NA, Meiser B, Butow PN, Soames Job RF, Mann GJ. Anticipated uptake of genetic testing for familial melanoma in an Australian sample: an exploratory study. Psychooncology. 2007 Jan;16(1):69-78.

48 Levin T, Mæhle L. Uptake of genetic counseling, genetic testing and surveillance in hereditary malignant melanoma (CDKN2A) in Norway. Fam Cancer. 2017 Apr;16(2):25765.

49 Taber JM, Aspinwall LG, Kohlmann W, Dow $\mathrm{R}$, Leachman SA. Parental preferences for CDKN2A/p16 testing of minors. Genet Med. 2010 Dec;12(12):823-38.

50 Aspinwall LG, Stump TK, Taber JM, Drummond DM, Kohlmann W, Champine M, et al. Genetic test reporting of CDKN2A provides informational and motivational benefits for managing melanoma risk. Transl Behav Med. 2018 Jan;8(1):29-43.

51 Buchanan AH, Voils CI, Schildkraut JM, Fine C, Horick NK, Marcom PK, et al. Adherence to Recommended Risk Management among Unaffected Women with a BRCA Mutation. J Genet Couns. 2017 Feb;26(1):79-92.
52 Milne S, Sheeran P, Orbell S. Prediction and Intervention in Health-Related Behavior: A Meta-Analytic Review of Protection Motivation Theory. J Appl Soc Psychol. 2000;30(1): 106-43.

53 Haga SB, Barry WT, Mills R, Svetkey L Suchindran S, Willard HF, et al. Impact of delivery models on understanding genomic risk for type 2 diabetes. Public Health Genomics. 2014;17(2):95-104.

54 Rothstein MA. Time to End the Use of Genetic Test Results in Life Insurance Underwriting. J Law Med Ethics. 2018 Sep;46(3): 794-801.

55 Biesecker BB, Ishibe N, Hadley DW, Giambarresi TR, Kase RG, Lerman C, et al. Psychosocial factors predicting BRCA1/BRCA2 testing decisions in members of hereditary breast and ovarian cancer families. Am J Med Genet. 2000 Aug;93(4):257-63.

56 Forde C, Brunstrom K, Woodward E, Bowers $\mathrm{N}$, Pereira M, Wallace AJ, et al. Uptake of presymptomatic testing for BRCA1 and BRCA2 is age, gender, offspring and time-dependent. J Med Genet. 2020 Apr;jmedgenet-2019106544.

57 Burns PB, Rohrich RJ, Chung KC. The levels of evidence and their role in evidence-based medicine. Plast Reconstr Surg. 2011 Jul; 128(1):305-10.

58 Aspinwall LG, Taber JM, Kohlmann W, Leaf SL, Leachman SA. Unaffected family members report improvements in daily routine sun protection 2 years following melanoma genetic testing. Genet Med. 2014 Nov; 16(11): $846-53$ 